Martínez Clares, P.; González Lorente, C. y Rebollo Quintela, N. (2019). Competencias para la empleabilidad: un modelo de ecuaciones estructurales en la Facultad de Educación. Revista de Investigación Educativa, 37(1), 57-73. DOI: http://dx.doi.org/10.6018/rie.37.1.343891

\title{
Competencias para la empleabilidad: un modelo de ecuaciones estructurales en la Facultad de Educación
}

\author{
Employability Skills:A Structural Equation Model at the \\ Faculty of Education
}

\author{
Pilar Martínez Clares*, Cristina González Lorente* y Nuria Rebollo Quintela** \\ *Universidad de Murcia \\ ** Universidade da Coruña
}

\begin{abstract}
Resumen
Ante la nueva Revolución 4.0, donde la concepción de la empleabilidad de los estudiantes va más allá de la adquisición de un empleo en particular, este trabajo profundiza en la valoración del universitario acerca del desarrollo de competencias para la empleabilidad que se produce durante su formación universitaria. Además, incluye una validación de constructo de la escala de competencias presentada mediante el AFC y se analizan posibles diferencias en función de las tres universidades españolas analizadas: Universidad de Murcia, Universidad de Granada y Universidade da Coruña. Con este objetivo, participan 830 estudiantes de último curso de Grado de la Facultad de Educación de dichas universidades mediante la cumplimentación del Cuestionario de Orientación e Inserción Laboral (COIL). Para el análisis de los datos se recurre a la estadística descriptiva e inferencial a través del programa estadístico SPSS v23 y al programa AMOS para realizar el Modelo de Ecuaciones Estructurales. Los resultados muestran unos índices de bondad de ajuste óptimos, que garantizan la adecuación del mismo a los datos empíricos; además, se comprueba que existe una tendencia común entre los estudiantes de la facultad de educación, la relativa al desarrollo medio-bajo de competencias
\end{abstract}

Correspondencia: Cristina González Lorente, c.gonzalezlorente@um.es, Departamento de Métodos de Investigación y Diagnóstico en Educación. Facultad de Educación. Universidad de Murcia. Campus de Espinardo, s/n. 30100 Murcia (España). 
para la empleabilidad, con diferencias significativas entre universidades. A partir de estos resultados, es preciso buscar sinergias de comunicación y cooperación entre el contexto productivo, los agentes sociales y la universidad, y hacerlo tanto local como nacionalmente, para encontrar el equilibrio y transferencia que exige el EEES en la nueva Revolución 4.0.

Palabras clave: competencia; educación superior; empleabilidad; inserción laboral; modelo de ecuaciones estructurales.

\begin{abstract}
In the face of the fourth industrial revolution, where the conception of the employability of students goes beyond the acquisition of a particular job, this research deepens the assessment of the university student about the development of skills for employability that occurs during their university education. In addition, it includes a construct validation of the competencies scale presented by the CFA and possible differences are analyzed according to the three Spanish universities analyzed: University of Murcia, University of Granada and University of A Coruña. With this objective, 830 final year students in the faculty of education of these universities participated by completing the Cuestionario de Orientación e Inserción Laboral (COIL). For the analysis of the data, descriptive and inferential statistics were used through the statistical program SPSS v23 and the AMOS program to carry out the Structural Equation Model. The results show optimal model fit indexes, which guarantee the suitability of this model with the empirical data. In addition, it is verified that there exists a common tendency among all students of the faculty of education relative to the medium-low development of competencies for employability, but with significant competency differences varying among each university. Based on these results, it is necessary to seek synergies of communication and cooperation among the job markets, the social agents, and the university, and to do it both locally and nationally in order to find the balance and transfer required by the EHEA in the fourth industrial revolution.

Keywords: skills; higher education; employability; entering the workforce; structural equation model.
\end{abstract}

\title{
Introducción
}

El mapa universitario del S. XXI demanda a la Educación Superior una formación transferible y competente en un contexto social y mercado laboral convulso, sometido a continuos cambios y cada vez más exigente y competitivo (do Ceu Taveira y Rodríguez Moreno, 2010). Un escenario que se va sobreponiendo a la celeridad que le imprime el mundo global y complejo en el que estamos inmersos, y que reclama por igual el dominio de competencias técnicas como el de competencias profesionales, aquellas que son transferibles, generalizables y demandadas en los diversos contextos socio laborales donde transitamos y que además, requieren de "habilidades analíticas y la capacidad de resolución de problemas, la capacidad de innovación y adaptabilidad ante el permanente cambio, así como habilidades comunicativas" (Alarcón, 2018, p. 147), es decir, del desarrollo y dominio de competencias transversales. 
La universidad, ya no sólo debe de responder a las exigencias del mercado laboral actual, sino que tiene que ser proactiva, anticipándose a las nuevas demandas de los empleos existentes y a los yacimientos de empleo emergentes (Pagés Serra y Ripani, 2017), y más aún en el futuro presente de la Revolución 4.0.

En este cambio de época o tal vez, época de cambios, nos debemos cuestionar que la empleabilidad de los estudiantes universitarios va más allá del empleo, pues esta debe contemplar, como ya señalaba la Organización Internacional del Trabajo (2004), las competencias y cualificaciones transferibles que refuerzan la capacidad de las personas para aprovechar las oportunidades de educación y de formación que se les presenten con miras a encontrar y conservar un trabajo decente, progresar en la empresa o cambiar de empleo, y adaptarse a la evolución de la tecnología y de las condiciones del mercado de trabajo.

Las instituciones de educación superior deben ser claro reflejo del enfoque competencial asumido por el común Espacio Europeo de Educación Superior (EEES), que apuesta por la promoción de la empleabilidad, facilitando el uso de "un lenguaje común para definir los perfiles académicos y profesionales permitiendo la comparación y comunicación entre los diferentes países" (Navaridas Nalda, Jiménez Trens y Fernández Ortiz, 2016, p. 338), buscando, a la vez, la mayor sinergia posible entre la formación inicial (formación universitaria) y las demandas del mercado laboral (Figuera, Raitz y LLanes, 2013; Llanes Ordoñez, Figuera Gazo y Torrado Fonseca, 2017), hallándose como indicador de calidad en los SIGC, la inserción laboral de sus egresados.

Una inquietud creciente y recurrente en las últimas décadas ha sido la definición y categorización de estas competencias, así como el análisis de las mismas (ClementeRicolfe y Escribá-Pérez, 2013; Gil Flores, 2007). Se hace necesario, precisar una definición común del término competencia, reconocida tanto en el ámbito académico como en el laboral debido a que persisten las discrepancias a la hora de delimitarla motivado por la multitud de interpretaciones, desde precisiones más restrictivas a más extensas. Estas últimas, tienen una visión más amplia que abarca tanto el conocimiento como las actitudes, habilidades y aspectos sociales y más personales y motivacionales (Gijbels, 2011).

El gran abanico de investigaciones realizadas permite reflejar las diferentes visiones de una misma realidad, aportando la perspectiva de los múltiples agentes implicados en el proceso; alumnado (Medina Rivilla, Domínguez Garrido y Sánchez Romero, 2013), egresados (Lluch Molins, Fernández-Ferrer, Pons Seguí y Cano García, 2017), profesorado (Corominas et al., 2006) y empresarios y agentes sociales (Alonso, Fernández y Nysen, 2009). Otros estudios apuestan por realizar una complementariedad entre varias visiones: alumnado y profesorado (Mir Acebrón, 2007) y empresas y titulados (Cajide et al., 2002; Freire, Teijeiro y Pais, 2011). En el horizonte de las organizaciones, son varios los trabajos realizados sobre competencias, entre otros, el Informe de Competencias Profesionales y Empleabilidad, del Consejo Económico y Social (CES, 2015), el Estudio de Posiciones y Competencias más Demandas en la Empresa 2017, que aporta la perspectiva de los responsables de recursos humanos (EPYCE, 2018), y el documento elaborado por la Comisión Económica para América Latina y el Caribe (ECLAC) de Naciones Unidas sobre la identificación y anticipación de los requisitos competenciales (Van Breugel, 2017). 
Ante un panorama educativo y sociolaboral como el referido, cobra vital importancia la formación en competencias; Detenerse en reflexionar esta realidad actual, permite dirimir en qué momento nos encontramos y hacia dónde debemos de avanzar, buscando un mayor engranaje entre las instituciones de educación superior y el mercado laboral y las necesidades que éste demanda. Esta realidad lleva a plantear esta investigación que persigue profundizar en las percepciones del alumnado universitario sobre las competencias profesionales adquiridas en su formación inicial y que pueden facilitar su proceso de inserción socio-laboral y favorecer su empleabilidad. En concreto, los objetivos definidos son: a) conocer y describir la percepción del alumnado sobre el desarrollo de las competencias para la empleabilidad durante su formación universitaria, b) realizar una validación de la consistencia interna de la escala de competencias a partir del análisis factorial confirmatorio y c) analizar las posibles diferencias en las covarianzas de dicha escala en función de la universidad de origen en la que se sitúa el estudiante.

\section{Método}

\section{Población y Muestra}

La muestra de esta investigación está conformada por un total de 830 estudiantes de último curso de Grado de la Facultad de Educación de tres universidades españolas: Universidad de Murcia, Universidad de Granada y Universidade da Coruña. La edad media de estos participantes es de 23 años (DT=3.744), con una mayor representación femenina (78.8\%) frente al alumnado masculino $(21.2 \%)$.

Para la obtención de esta muestra, se realiza un muestreo no probabilístico por conglomerados, con un nivel de confianza de $99 \%$ y un error muestral de $4 \%$. La muestra es representativa de los estudiantes de último curso de grado en los títulos de la facultad de educación analizados cuya distribución, por motivos de acceso, queda representada como se resume en la tabla 1.

Tabla 1

Distribución de la muestra por universidad y grado

\begin{tabular}{lcccccccc}
\hline & \multicolumn{2}{c}{$\begin{array}{c}\text { Universidad de } \\
\text { Murcia (UM) }\end{array}$} & \multicolumn{2}{c}{$\begin{array}{c}\text { Universidad de } \\
\text { Granada (UGR) }\end{array}$} & $\begin{array}{c}\text { Universidade da } \\
\text { Coruña (UDC) }\end{array}$ & \multicolumn{2}{c}{ Total } \\
\cline { 2 - 10 } & $\mathbf{N}$ & $\mathbf{\%}$ & $\mathbf{N}$ & $\mathbf{\%}$ & $\mathbf{N}$ & $\mathbf{\%}$ & $\mathbf{N}$ & $\%$ \\
\hline Educación Primaria & 103 & 12.41 & 202 & 24.34 & 103 & 12.41 & 408 & 49.16 \\
Educación Infantil & 125 & 15.06 & 0 & 0 & 91 & 10.96 & 216 & 26.02 \\
Pedagogía & 92 & 11.08 & 32 & 3.86 & 0 & 0 & 124 & 14.94 \\
Educación Social & 41 & 4.94 & 41 & 4.94 & 0 & 0 & 82 & 9.88 \\
Total & 361 & 43.49 & 275 & 33.13 & 194 & 23.37 & 830 & 100 \\
\hline
\end{tabular}




\section{Instrumento}

Como instrumento de recogida de información se utiliza el Cuestionario de Orientación e Intermediación Laboral (COIL), diseñado para una investigación más amplia de la que forma parte este trabajo. A partir de un análisis de validez de contenido y consistencia interna del mismo (Autor y autor, 2018), éste queda estructurado en cinco grandes bloques que abordan el proceso de inserción socio-laboral del universitario:

- datos personales y académicos,

- experiencia académico-profesional,

- formación con el Grado y satisfacción,

- expectativas y creencias hacia el empleo,

- recursos y servicios que pueden facilitar el proceso de inserción socio-laboral.

Para la realización de este trabajo de investigación en particular, se extrae la información únicamente del tercer bloque, en el cual se incluye la escala de competencias para la inserción laboral objeto de estudio, con la valoración del desarrollo de dichas competencias durante el Grado por parte del alumnado en la escala tipo Likert de 1 a 5 (donde $1=$ nada y $5=$ mucho).

\section{Procedimiento}

Tras una búsqueda bibliográfica en profundidad sobre la inserción socio-laboral de personas con titulaciones de educación superior, se concretan los objetivos de la investigación más amplia de la que forma parte este trabajo. A continuación, se procede al diseño del cuestionario COIL, donde se incluye la escala de competencias para la empleabilidad que se recoge en la tabla 2.

Tabla 2

Mapa funcional del modelo teórico de competencias profesionales para la inserción socio-laboral

\begin{tabular}{lll}
\hline Competencia & \multicolumn{1}{c}{ Elemento de competencia } & \multicolumn{1}{c}{ Código } \\
\hline \multirow{4}{*}{$\begin{array}{c}\text { Competencias } \\
\text { participativas }\end{array}$} & Flexibilidad y orientación al cambio & D_5.10 \\
& Toma de decisiones & D_5.6 \\
& Motivación por el logro & D_5.11 \\
& Organización y planificación & D_5.3 \\
& Responsabilidad de aprendizaje y adaptación & D_5.9 \\
& Análisis, síntesis y crítica & D_5.5 \\
& Trabajo en equipo & D_5.2 \\
& Compromiso con la organización & D_5.8 \\
& Orientación a las personas & D_5.12 \\
\hline
\end{tabular}




\begin{tabular}{cll}
\hline & Búsqueda de la excelencia & D_5.15 \\
& Capacidad de resiliencia y tolerancia a la frustración & D_5.19 \\
& Capacidad para trabajar bajo presión & D_5.13 \\
Competencias & Habilidades de comunicación & D_5.4 \\
personales & Conciencia de uno mismo & D_5.1 \\
& Innovación & D_5.16 \\
& Emprendimiento & D_5.17 \\
& Liderazgo & D_5.18 \\
& Resolución de conflictos y técnicas de negociación & D_5.14 \\
\hline
\end{tabular}

Tras su diseño, el cuestionario COIL se somete a un exhaustivo procedimiento de validación y pilotaje. Seguidamente, se contacta con el profesorado de $4^{\circ}$ curso de Grado de las tres universidades que participan en el estudio para acordar el horario de clase más adecuado para la recogida de información, asegurando el carácter voluntario y confidencialidad de los datos recabados que, posteriormente, se analizan con la ayuda del programa estadístico SPSS v23 y AMOS v7. Por último, se presentan las principales conclusiones, limitaciones y prospectivas que dan forma a este trabajo.

\section{Análisis de datos}

El análisis estadístico de los datos permite obtener la consistencia interna de la escala de competencias para la inserción socio-laboral de los estudiantes de las titulaciones de educación en las distintas universidades analizadas. En primer lugar, se realiza un análisis descriptivo con el cálculo de la media y desviación típica de cada elemento de competencia, tanto para la muestra global como por universidad. Estos datos permiten contrastar a su vez, las posibles diferencias en el desarrollo de dichas competencias durante la formación en función de la universidad; un análisis que se completa con la estadística inferencial y la prueba de Kruskal-Wallis, al no asumirse normalidad en los datos pero sí, una misma distribución de la muestra. En este caso, se asume como nivel de significatividad $p \leq 0.05$.

A continuación, se realiza el cálculo del coeficiente del Alfa de Cronbach, la homogeneidad de los datos y el índice de KMO junto con la prueba de esfericidad de Bartlett. Tras comprobar la aplicabilidad del análisis factorial con un nivel de significatividad de $p \leq .05$ en la prueba de Bartlett, se acepta la hipótesis nula $\left(\mathrm{H}_{0}\right)$ y el modelo de competencias se considera óptimo para aplicar el Análisis Factorial Confirmatorio (AFC). Dicho análisis viene delimitado por los índices de bondad de ajuste del modelo, cuya idoneidad se evalúa en un rango de valores entre 0 y 1; En el caso de los índices de ajuste comparativo, como el CFI o IFI, se consideran valores adecuados $\mathrm{y}$, por tanto, de ajuste al modelo, cuando son superiores a 0.8 , mientras que para los índices de ajuste de parsimonia como el RMSEA, el valor de referencia debe ser igual o inferior a 0.05 (Schumacker y Lomax, 2004). Una vez comprobado el ajuste estadístico del modelo de competencias, se examina su invariancia a través del modelo estructural multigrupo, cuya variable de agrupación es 
la Universidad de origen. Este análisis se focaliza tanto en las variaciones de los pesos de regresión como en los índices de bondad de ajuste utilizados previamente.

\section{Resultados}

Antes de realizar el AFC, se obtienen los resultados para comprobar la consistencia interna o fiabilidad de la escala de competencias para la inserción socio-laboral. El estadístico Alfa de Cronbach $(\alpha)$, muestra unos valores satisfactorios tanto en la escala global $(\alpha=.902)$ como en las dos sub-escalas presentadas en el gráfico de sedimentación de la Figura 1 (.830 y .560 para el primer y segundo factor, respectivamente). Esta primera aproximación a través del Análisis Factorial Exploratorio (AFE) confirma así, la existencia de 2 factores que explican el $43.78 \%$ de la varianza. Además, con la prueba de esfericidad de Bartlett, se comprueba que el nivel de significatividad es inferior a .05 , con un valor de Chi-cuadrado de $4357,340(\mathrm{gl}=171)$ y de .943 para el valor del índice de muestra de Kaiser- Meyer-Olkin (KMO).

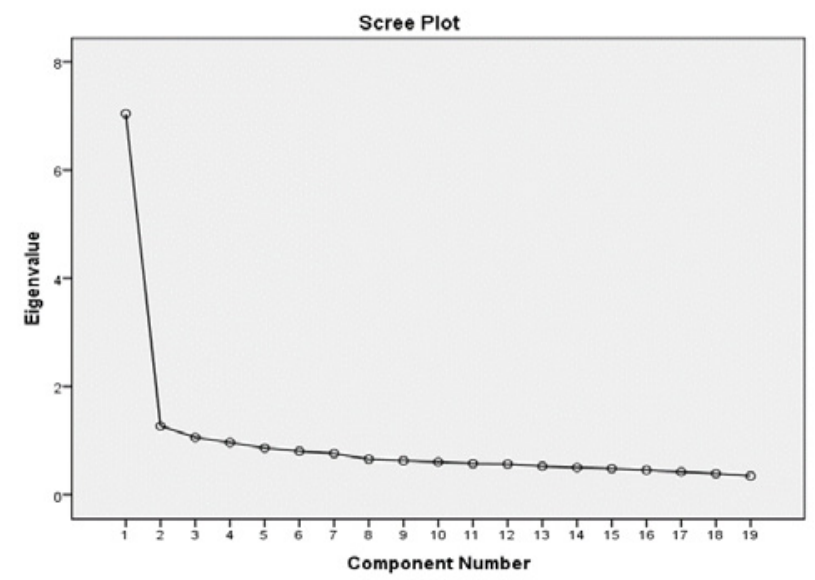

Figura 1. Gráfico de sedimentación para la escala de competencias

Respecto al análisis descriptivo del desarrollo de cada uno de estos elementos de competencia, la tabla 3 recoge la media y desviación típica para cada uno de ellos, clasificados por universidad, así como la prueba de Kruskal-Wallis para medir posibles diferencias significativas. En primer lugar, destaca la elevada media del elemento de competencia D_5.8 “Trabajo en equipo y cooperación” () que junto con D_5.5 “Responsabilidad y perseverancia" () son las que más se desarrollan dentro del aula universitaria desde la visión del alumnado. En cambio, "Liderazgo" (D_5.18), "Emprendimiento" (D_5.19) y "Resiliencia y tolerancia a la frustración" (D_5.17), con una media próxima a 3.20, se corresponden con las competencias menos trabajadas a lo largo de los estudios universitarios.

En general las puntuaciones aportadas por los estudiantes de la UM son más elevadas que el resto, mientras que el alumnado de la UGR parece menos optimista en 
sus valoraciones, con unas medias en el desarrollo de las competencias durante su formación inicial que oscilan en torno al valor 3 de la escala (tabla 3). En todas ellas se sigue la tendencia media destacada anteriormente, aunque en el caso de las primeras competencias relacionadas con la "conciencia de uno mismo", las "habilidades de comunicación" o la "organización y planificación", junto con el "trabajo en equipo", los estudiantes de la UM superan de forma destacada el punto medio para el total de participantes. En el caso de los estudiantes de la UDC, superan esta media cuando valoran el desarrollo de la "búsqueda de la excelencia" (D_5.13), quedándose más alejados en relación a las competencias personales de "innovación", "emprendimiento", "liderazgo" o "resiliencia".

Tabla 3

Desarrollo de unidades de competencias por universidad. Media, desviación típica y prueba de KruskalWallis

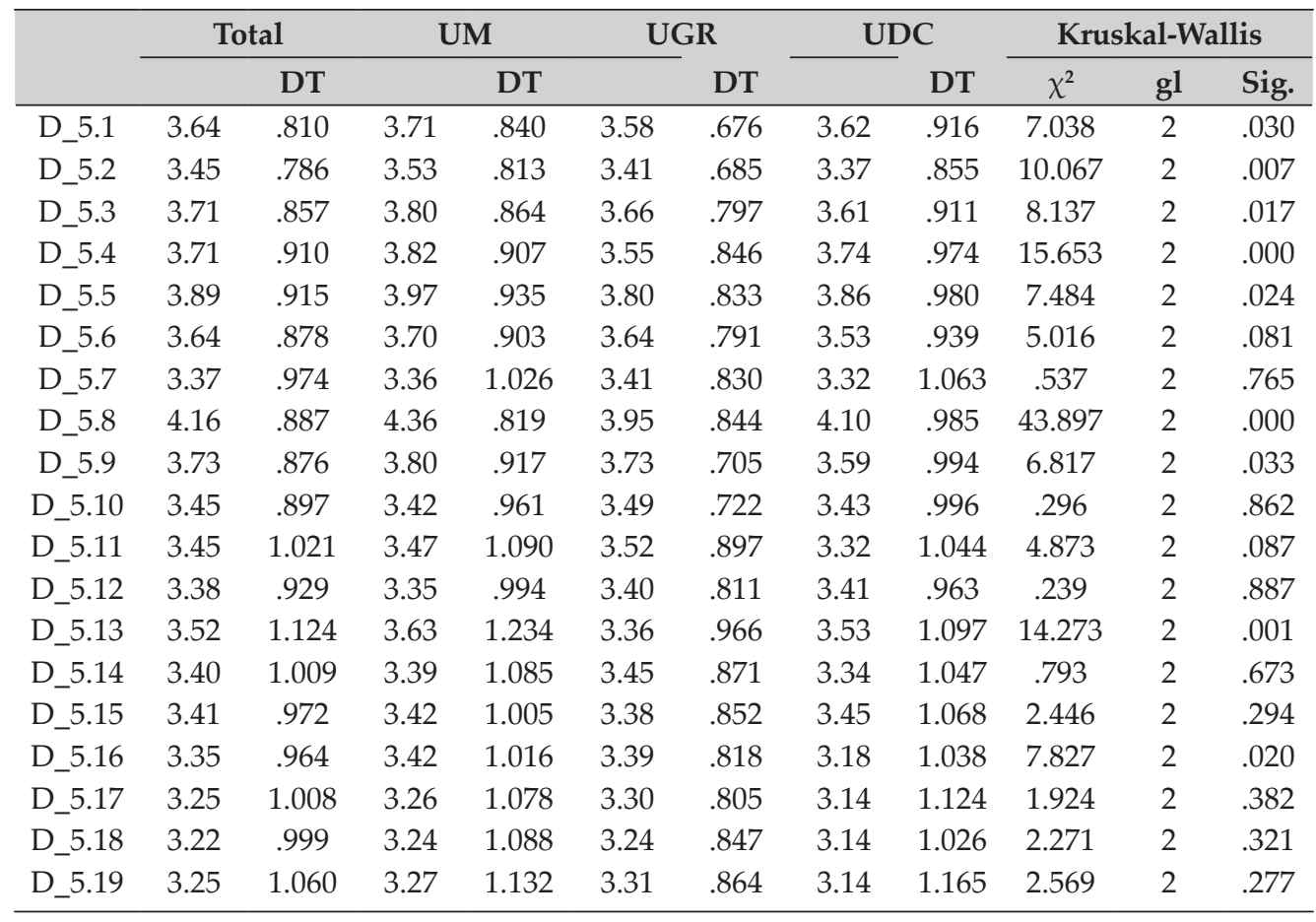

Como refleja la tabla 3, existen diferencias significativas en 9 de los ítems analizados, de forma que competencias como el "trabajo en equipo" presentan medias dispares entre sí, aun siendo la más desarrollada en las tres universidades. Esta diferencia viene acompañada de las unidades de competencia personal "habilidades para la comunicación" y "trabajar bajo presión" que con un valor de $p$ de .000 y .001, respectivamente, son valoradas de forma distinta por el alumnado de las universidades 
participantes. En estos casos, son los estudiantes de la UM los que mejor valoran dichas competencias, con medias superiores al resto de participantes de la UGR y UDC.

Pese a estas variaciones en la media del desarrollo de estos 19 elementos de competencia, se opta por realizar un análisis factorial confirmatorio (AFC) sin necesidad de restructurar el modelo de competencias presentado ni eliminar alguno de sus ítems, ya que, en tal caso, el coeficiente Alfa de Cronbach disminuye. Dada la distribución normal de las variables estudiadas, se recurre al método de Máxima Verosimilitud (ML) para realizar el AFC. Desde el ML, se plantea el modelo de medida que se representa en la figura 2 , donde se indican las relaciones de las dos variables latentes (Competencias personales y participativas) con sus respectivas variables observadas y que hacen referencia, a las unidades de competencia valoradas por el alumnado con respecto al desarrollo de las mismas durante su formación universitaria. Asimismo, se puede distinguir la covarianza entre las dos variables latentes (relacionadas entre sí por una flecha bidireccional) que permite contrastar la idoneidad de los indicadores en la medición de dichas variables.

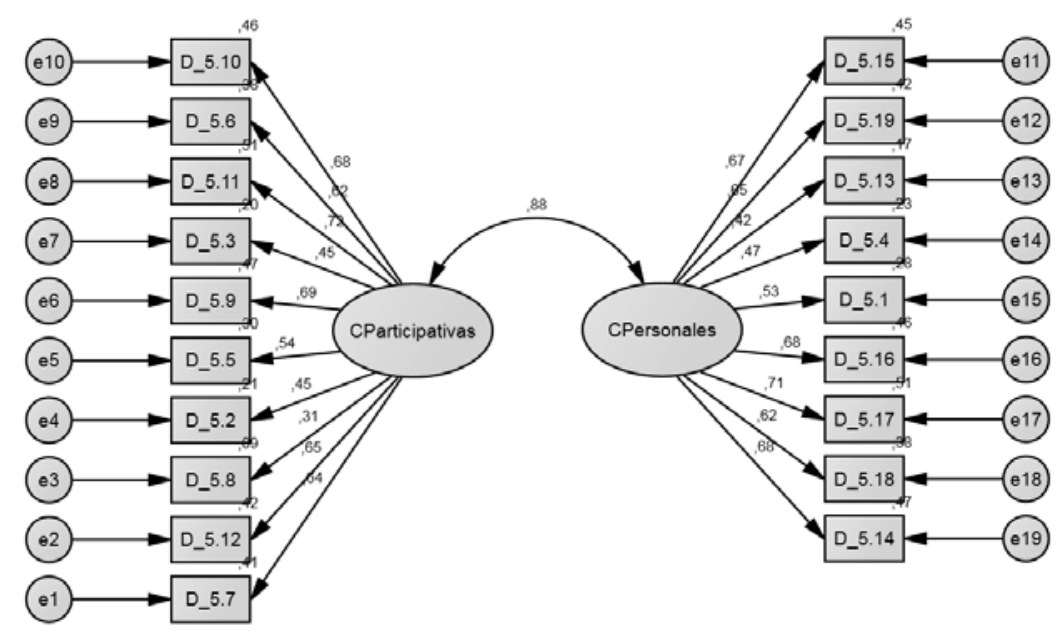

Figura 2. Modelo de ecuaciones estructurales de la escala de competencias para la inserción socio-laboral del universitario

La representación gráfica refleja los pesos de regresión entre las dos variables latentes (competencias participativas y personales) y el conjunto de las variables observables. En general, se observan valores superiores a .50 en su varianza o muy próximos a este valor, con la excepción del elemento de la competencia participativa "trabajo en equipo y cooperación" (D_5.8). En los 19 elementos de competencia analizados, la relación que se establece con sus respectivas variables latentes es significativa en todos los casos ( $p$ $=.000)$, con una covarianza alta (.88).

Para hacer una aproximación más ajustada al modelo presentado, se realiza un diagnóstico de la bondad de ajuste del modelo a partir de la interpretación de los índices más utilizados para la aceptación o rechazo del modelo. Debido al tamaño de 
la muestra (superior a 200 sujetos) el valor del estadístico Chi-Cuadrado () y su nivel de significatividad no se tienen en consideración por el alto nivel de influencia que tiene la muestra en dicho estadístico. Por esta razón, este análisis se centra en índices de medidas incrementales de ajuste como el índice de ajuste normado (NFI), y el índice de ajuste comparativo (CFI). Ambos índices se comparan con el estadístico y su valor oscila entre 0 y 1, siendo 1 el ajuste perfecto. Para el modelo analizado, estos índices se muestran próximos a la unidad (CFI=.920; $\mathrm{IFI=.921;} \mathrm{NFI=.889)} \mathrm{y} \mathrm{por} \mathrm{tanto,} \mathrm{revelan} \mathrm{la}$ adecuación del modelo a la realidad observada. No obstante, dichos datos se completan con el Índice de parsimonia RMSEA, que con un valor de .057, se sitúa en los niveles óptimos según Hu y Bentler (1999) para que el ajuste sea aceptable.

Este ajuste del modelo se mantiene al realizar el análisis de forma separada para cada uno de los subgrupos entre la UM, la UGR y la UDC. Sin embargo, aparecen un mayor número de diferencias en este análisis multigrupo, cuando se presta atención a la relación que se establece entre las variables observables y latentes. En función de la Universidad de los estudiantes participantes (tabla 4) se representan los pesos estandarizados de regresión (P.E.R.) entre las variables observables, junto con el error estándar (S. E.) y el nivel de significatividad ( $p$ ).

Tabla 4

Peso de regresión (P.R.) y pesos estandarizados de regresión (P.E.R.) entre las variables observables

\begin{tabular}{|c|c|c|c|c|c|c|c|c|c|c|c|}
\hline & & & & UM & & & UGR & & & JDC & \\
\hline & & & S.E. & $p$ & P.E.R. & S.E. & $p$ & P.E.R. & S.E. & $p$ & P.E.R. \\
\hline D_5.7 & $<--$ & CPart. & & $* * *$ & .721 & & $* * *$ & .533 & .073 & $* * *$ & .586 \\
\hline D_5.12 & $<--$ & CPart. & .082 & $* * *$ & .664 & .166 & $* * *$ & .534 & .067 & $* * *$ & .692 \\
\hline D_5.8 & $<--$ & CPart. & .063 & $* * *$ & .321 & .153 & $* * *$ & .344 & .069 & $* * *$ & .282 \\
\hline D_5.2 & $<--$ & CPart. & .070 & $* * *$ & .514 & .120 & $* * *$ & .330 & .060 & $* * *$ & .425 \\
\hline D_5.5 & $<--$ & CPart. & .076 & $* * *$ & .506 & .177 & $* * *$ & .566 & .068 & $* * *$ & .599 \\
\hline D_5.9 & $<-$ & CPart. & .079 & $* * *$ & .738 & .144 & $* * *$ & .565 & .069 & $* * *$ & .668 \\
\hline D_5.3 & $<--$ & CPart. & .071 & $* * *$ & .394 & .168 & $* * *$ & .484 & .063 & $* * *$ & .491 \\
\hline D_5.11 & $<--$ & CPart. & .091 & $* * *$ & .790 & .198 & $* * *$ & .632 & .072 & $* * *$ & .673 \\
\hline D_5.6 & $<-$ & CPart. & .075 & $* * *$ & .606 & .175 & $* * *$ & .604 & .065 & $* * *$ & .650 \\
\hline D_5.10 & $<--$ & CPart. & .080 & $* * *$ & .730 & .153 & $* * *$ & .542 & .070 & $* * *$ & .680 \\
\hline D_5.15 & $<--$ & CPers. & & $* * *$ & .715 & & $* * *$ & .610 & .074 & $* * *$ & .659 \\
\hline D_5.19 & $<--$ & CPers. & .100 & $* * *$ & .694 & .139 & $* * *$ & .481 & .082 & $* * *$ & .662 \\
\hline D_5.13 & $<--$ & CPers. & .110 & $* * *$ & .451 & .152 & $* * *$ & .342 & .076 & $* * *$ & .404 \\
\hline D_5.4 & $<--$ & CPers. & .080 & $* * *$ & .439 & .135 & $* * *$ & .494 & .067 & $* * *$ & .523 \\
\hline D_5.1 & $<--$ & CPers. & .076 & $* * *$ & .498 & .110 & $* * *$ & .554 & .064 & $* * *$ & .553 \\
\hline D_5.16 & $<--$ & CPers. & .091 & $* * *$ & .709 & .142 & $* * *$ & .528 & .072 & $* * *$ & .725 \\
\hline D_5.17 & $<--$ & CPers. & .098 & $* * *$ & .771 & .133 & $* * *$ & .491 & .078 & $* * *$ & .752 \\
\hline D_5.18 & $<--$ & CPers. & .097 & $* * *$ & .629 & .138 & $* * *$ & .584 & .071 & $* * *$ & .629 \\
\hline D_5.14 & $<--$ & CPers. & .098 & $* * *$ & .721 & .156 & $* * *$ & .680 & .072 & $* * *$ & .644 \\
\hline
\end{tabular}

Nota: S.E.=Estimación del error; C.R.=Ratio Crítico; $p=$ nivel de significatividad $\left({ }^{* * *}=.005\right)$. 
De forma generalizada los pesos de regresión son mayores a .50, destacando la variación que existe cuando se analiza el elemento de competencia "Innovación" (D_5.16) y "Emprendimiento" (D_5.17) cuya varianza con la competencia personal es elevada en la UM (.709 y .771) y UCD (.725 y .752), mientras que en la UGR apenas alcanza dicho valor. Igualmente, es reseñable que los P.E.R. son superiores entre la valoración realizada en la UM, donde existe una mayor relación entre la D_5.7 (“Orientación a las personas") o D_5.11 ("Motivación por el logro") con la competencia participativa. Entre los datos de la UDC, los P.E.R. presentan valores ligeramente inferiores, mostrando una varianza entre los elementos que constituyen las dos competencias analizadas (participativas y personales) en torno a .6. Por su parte, los datos pertenecientes a la UGR reflejan unos valores inferiores con respecto al resto situándose en torno a .5 , mientras que en aquellos casos en los que este valor es inferior, coinciden con los pesos de regresión más bajos entre la UM y UDC. En cualquier caso, la relación entre las variables observables y latentes es significativa $(p=.000)$ en los tres subgrupos analizados.

Estas diferencias significativas también aparecen entre las dos variables latentes de forma que, independientemente de la universidad de los participantes, este análisis muestra los elevados P.E.R. que mantienen las competencias personales y participativas en el modelo de competencias para la inserción socio-laboral de los universitarios (tabla 5). En todos los subgrupos, así como en el estudio del modelo para el total de la muestra, los pesos de regresión están próximos a la unidad, destacando especialmente la UDC que, con un P.E.R.=.931, es la universidad donde más fuerza presenta dicha relación.

Tabla 5

Peso de regresión (P. R.) y pesos estandarizados de regresión (P.E.R.) entre las variables latentes

\begin{tabular}{lcccccccc}
\hline \multicolumn{2}{c}{ Relación entre variables } & Univ. & Estimate & S.E. & C.R. & $p$ & Estimate \\
\hline CPers. & $<-->$ & CPart. & Total & .364 & .033 & 11.162 & $* * *$ & .865 \\
CPers. & $<-->$ & CPart. & UM & .485 & .063 & 7.679 & $* * *$ & .865 \\
CPers. & $<-->$ & CPart. & UGR & .184 & .036 & 5.166 & $* * *$ & .858 \\
CPers. & $<-->$ & CPart. & UDC & .386 & .066 & 5.877 & $* * *$ & .913 \\
\hline
\end{tabular}

Nota: S.E.=Estimación del error; C.R.=Ratio Crítico; $p=$ nivel de significatividad $(* * *=.005)$.

\section{Discusión y conclusiones}

En el actual escenario económico, social y laboral, queda justificada la importancia de acercar posturas y crear puentes de entendimiento y cooperación entre las instituciones de educación superior y el mercado laboral. El centro de actuación se debe situar en el estudiante, el futuro profesional del siglo XXI, es él quien debe articular desde su propio proyecto profesional y vital, un conjunto de competencias de acción profesional adquiridas durante la formación superior, para incrementar así, sus oportunidades en el proceso de transición a la vida activa (Llanes Ordoñez et al., 2017; Van der Heijde, 2014). 
Con la intención de contribuir en la mejora del conocimiento de las competencias que se desarrollan para favorecer el proceso de inserción socio-laboral desde la formación universitaria, esta investigación ha profundizado en esta dirección, deteniéndose en las percepciones y valoraciones del alumnado de la facultad de educación de tres universidades de nuestro contexto y analizando paralelamente la validez del modelo de competencias propuesto a nivel teórico y las posibles diferencias entre los estudiantes participantes.

Los resultados manifiestan una tendencia común entre los estudiantes de la facultad de educación, la relativa al desarrollo medio-bajo de competencias para la empleabilidad. Estos datos se repiten entre estudiantes de diferentes ramas del conocimiento y titulaciones, también referidas en otros estudios como transversales o genéricas (Michavila, Martínez, Martín-González, García-Peñalvo, y Cruz-Benito, 2016; Villardón, 2015). Recientemente, Bozu (2017) señala que los futuros profesionales del ámbito educativo demandan una formación más integral con un mayor desarrollo de competencias para acceder a un empleo, más allá de la especialización profesional.

En los participantes, no sólo este desarrollo alcanza un valor medio, sino que, además, existen ciertas diferencias en el nivel con el que se trabajan las competencias dentro de la formación universitaria. Si bien el "trabajo en equipo" junto con la "responsabilidad y perseverancia" son las competencias más desarrolladas con un valor próximo a 4 en esta misma escala o incluso superior, estos resultados contrastan con otras competencias como el "liderazgo", el "emprendimiento" o la "resiliencia y tolerancia a la frustración", cuya percepción respecto a su aplicación en el aula durante los diferentes grados de la facultad de educación es, cuanto menos, mejorable.

Estos datos están en concordancia con el estudio de Lluch Molins et al. (2017), donde las valoraciones de los estudiantes insisten en la fuerte aportación que realiza la universidad en el desarrollo de la competencia de trabajo en equipo en contraposición al emprendimiento y la competencia de ética y sostenibilidad, que es menos desarrollada. Diferentes estudios destacan la importancia de estas competencias junto con la capacidad de innovación y adaptabilidad ante el permanente cambio, como imprescindibles para que los egresados sean competencialmente sostenibles y puedan obtener mejores perspectivas y oportunidades en su proceso de inserción socio-laboral (Alarcón, 2018; Rodríguez Gómez, Ibarra Saiz y Cubero Ibáñez, 2018).

Cuando se les pregunta a los empleadores (Freire et al., 2011; Montoro, Mora y Ortíz, 2012), éstos valoran la capacidad de aprendizaje autónomo y de adaptación a nuevas situaciones, la creatividad, el liderazgo, la iniciativa y el espíritu emprendedor, como las principales competencias que los universitarios deben poseer en un grado alto de desarrollo al término de su formación inicial, para acceder al mercado laboral con un adecuado nivel de empleabilidad. Por lo tanto, es pertinente y necesario como recomienda la OCDE (2015), alcanzar en España, un mayor equilibrio entre la oferta y la demanda actual de competencias, para garantizar desde la educación superior, que los egresados adquieran un alto nivel de competencias profesionales relevantes para la nueva realidad socioeconómica y laboral.

Asimismo, los resultados han evidenciado diferencias en la valoración del desarrollo competencial del alumnado en función de la universidad en la que cursan sus estudios. Aunque profundizar en el motivo puede ser objeto de futuras líneas de investigación, 
este dato puede estar relacionado, "con las tareas de aprendizaje y evaluación que realizan los estudiantes, con la retroalimentación efectiva que reciben durante el proceso o con su propia participación e implicación en el proceso de evaluación de competencias" (Rodríguez Gómez et al., 2018, p. 199). En cualquier caso, es importante buscar sinergias de comunicación y cooperación entre el contexto productivo, los agentes sociales y la universidad, y hacerlo tanto local como nacionalmente, para encontrar el equilibrio y transferencia que exige el EEES.

En esta línea, el modelo teórico de competencias presentado revela unos índices de bondad de ajuste elevados en los resultados obtenidos, todos ellos próximos a la unidad. Por lo tanto, existe una adecuación de la escala al modelo de ecuaciones estructurales propuesto, indicando que las variables observables y evaluadas por el alumnado (unidades de competencia) mantienen una fuerte relación entre sí, mientras que miden, de forma satisfactoria, las dos competencias planteadas como esenciales para favorecer la entrada del universitario al mercado laboral, competencias personales y participativas. Actualmente, son este conjunto de competencias que van más allá de un ámbito disciplinar en particular, las que facilitan en mayor medida, alcanzar mayores cotas de empleabilidad y con ello, favorecer el proceso de inserción socio-laboral del universitario (Medina y Jaruta, 2013).

Este ajuste del modelo se mantiene al realizar el análisis de forma separada para cada uno de los subgrupos de las universidades participantes. En todos ellos, los pesos de regresión entre las competencias participativas y personales están próximos a la unidad. Este hecho puede interpretarse a partir de la fuerte complementariedad que presentan las competencias participativas y personales (Autor y González Morga, 2018), es decir, aquellas que ponen en relación al trabajador con su entorno y enfatizan la eficacia y valor de la persona, cuya relación es difícil de desligar para afrontar el proceso de inserción socio-laboral.

Sin embargo, aparecen un mayor número de diferencias en este análisis por universidad cuando se presta atención a la carga o peso factorial que se establece entre las variables observables y latentes. Los datos de la UGR reflejan unos pesos de regresión inferiores con respecto al resto de universidades y sus valores se sitúan en torno a .5, mientras que en aquellos casos en los que este valor es inferior, coinciden con los pesos de regresión más bajos entre la UM y UDC. Diferencias que a la vista de los cambios acaecidos a raíz de la creación del espacio de convergencia europea y materializado en el EEES, no deberían estar presentes. Si bien la valoración que el alumnado hace del desarrollo de las competencias puede estar mediatizado por factores contextuales, sociales o de logro en el ámbito académico (Baartman y Ruijs, 2011), desde las instituciones de educación superior se debe dar un paso al frente y apostar, como señalan Navaridas Nalda et al. (2016), por la promoción de la empleabilidad a través de un lenguaje común que defina los perfiles académicos y profesionales, permitiendo la comparación y comunicación entre los diferentes países, pero también dentro de las propias universidades a nivel nacional.

Una de las limitaciones del estudio puede apreciarse en la diferencia de la muestra por titulaciones dentro de la facultad de educación, debido principalmente, a la falta de acceso por parte de los investigadores. Aunque es cierto que la muestra es elevada para realizar el tipo de análisis propuesto, así como equilibrada entre las tres universi- 
dades participantes, sería conveniente continuar en futuros estudios con la validación de la escala y análisis multigrupo entre titulaciones con datos más equitativos en una misma facultad para comprobar así, el comportamiento y variación en el desarrollo de competencias entre los diferentes grados de la Facultad de Educación.

Teniendo en cuenta todas estas aportaciones, junto con las líneas de prospección y limitaciones del estudio, queda patente que la formación inicial de los futuros egresados universitarios debe continuar la línea propuesta por el EEES, a partir de una mejora en el proceso de enseñanza-aprendizaje que aporte realmente, las competencias esenciales para una adecuada transición a la vida activa de los universitarios. Es preciso y pertinente enfocar su empleabilidad más allá de la adquisición de un empleo en particular en un contexto laboral que demanda grandes cotas de autonomía, flexibilidad, pensamiento crítico e innovación. En la misma línea que plantea Cabrera, Portillo y Prades (2016), se debe institucionalizar y generalizar entre la docencia universitaria la imperiosa necesidad de alcanzar un mejor equilibrio entre la enseñanza que se ofrece a los estudiantes y los resultados del aprendizaje competencial del egresado.

En el ámbito de la facultad de educación, la formación de los futuros profesionales debería incidir más en aspectos vinculados a la innovación y en las relaciones interpersonales dentro y fuera del aula, para en última instancia, aunar las lógicas académica y laboral, al mismo tiempo que se revisa de forma conjunta, cuál debe ser la misión de la universidad en la actualidad y a qué perfiles competenciales necesita dar respuesta (Lluch Molins et al., 2017).

\section{Referencias}

Alarcón, R. (2018). La formación para el trabajo y el paradigma de formación por competencias. Calidad en la educación, 0(16). Recuperado de https://www.calidadenlaeducacion.cl/index.php/rce/article/view/434

Alonso, L.E., Fernández, C. J., \& Nysen, J. (2009). El debate sobre las competencias. Una investigación cualitativa en torno a la educación superior y el mercado de trabajo en España. Madrid:ANECA

Alonso-Martín, P. (2010). La valoración de la importancia de las competencias transversales:comparación de su percepción al inicio y final de curso en alumnos de psicología. Revista de Investigación Educativa, 28 (1), 119- 140. Recuperado de http://revistas.um.es/rie/article/view/97821/109391

Baartman, L., \& Ruijs, L. (2011). Comparing students' perceived and actual competence in higher vocational education. Assessment an Evaluation in Higher Education, 36, 387.

Bozu, Z., \& Aránega, S. (2017). La formación inicial de maestros y maestras a debate: ¿Qué nos dicen sus protagonistas? Profesorado: Revista de currículum y formación del profesorado, 21(1), 143-163.

Bunk, G. (1994). La transmisión de las competencias en la formación y perfeccionamiento profesionales de la RFA. Revista Europea Formación Profesional, (1), 8-14.

Cabrera, N., Portillo, M.C.,\&Prades, A. (2016). Las competencias de los graduados universitarios y su evaluación. La perspectiva de los empleadores. En E. Cano, \& M. Fernández, M. (eds.). Evaluación por competencias: la perspectiva de las primeras promociones de graduados en el EEES (pp. 95-112.). Barcelona: Octaedro 
Cajide, J., Porto, A., Abeal, C., Barreiro, F., Zamora, E. Expósito, A., \& Mosteiro, J. (2002). Competencias adquiridas en la universidad y habilidades requeridas por los empresarios. Revista de investigación educativa, 20(2), 449-467. Recuperado de http:// revistas.um.es/rie/article/view/99011/94601

Clemente-Ricolfe, J.S., \& Escribá-Pérez, C. (2013). Análisis de la percepción de las competencias genéricas adquiridas en la universidad. Revista de educación, 362,535-561. Doi: 10.4438/1988-592X-RE-2013-362-241

Consejo Económico y Social (2015). Informe de competencias profesionales y empleabilidad. Recuperado de http://www.ces.es/documents/10180/2471861/Inf0315.pdf

Corominas, E., Tesouro, M., Capell, D., Teixidó, J., Pèlach, J., \& Cortada, R. (2006). Percepciones del profesorado ante la incorporación de las competencias genéricas en la formación universitaria. Revista de Educación, 341, 301-336. Recuperado de http:// www.revistaeducacion.mec.es/re341/re341_14.pdf

Do Ceu Taveira, M. , \& Rodríguez Moreno, M.L (2010). La gestión personal de la carrera y el papel de la orientación profesional. Teoría, práctica y aportaciones empíricas. Revista española de orientación y psicopedagogía, 21 (2), 335-345. Recuperado de http:// revistas.uned.es/index.php/reop/article/view/11536/11012

EPYCE (2018). Posiciones y Competencias más Demandas. Recuperado de http://marketing. eae.es/prensa/_EPyCE2017.pdf

Figuera, P., Raitz, T., \& Llanes, J. (2013). Os sentidos de formaçao acadêmica e do trabalho para estudantes de mestrado no Brasil e na Espanha. Educaçao tematica digital, 15 (3), 612-627. Recuperado de https://dialnet.unirioja.es/servlet/articulo?codigo $=4856196$

Freire, M. J., Teijeiro, M. M., y Pais, C. (2011). Políticas educativas y empleabilidad: ¿cuáles son las competencias más influyentes? Education Policy Analysis Archives, 19, 1-24.

Freire, M. J., Teijeiro, M. M., \& Pais, C. (2013). La adecuación entre las competencias adquiridas por los graduados y las requeridas por los empresarios. Revista de Educación, 362, 13-41.

Gijbels, D. (2011) Assessment of vocational competence in higher education: reflections and prospects. Assessment $\mathcal{E}$ Evaluation in Higher Education, 36 (4), 381-383, DOI: 10.1080/02602938.2011.581859

Gil Flores, J. (2007). La evaluación de competencias profesionales. Educación XXI, 10, 83-106.

Gómez Ruíz, M.A., Rodríguez Gómez, G., \& Ibarra Sáiz, M.S. (2013). COMPES: Autoinforme sobre las competencias básicas relacionadas con la evaluación de los estudiantes universitarios. Revista Española de Orientación Psicopedagógica, 24, 197-224. Recuperado de https://www.unav.edu/publicaciones/revistas/index.php/ estudios-sobre-educacion/article/view/2031/1896

Lara, A. (2014). Introducción a las ecuaciones estructurales en AMOS y $R$ (Tesis de Máster). Granada: Universidad de Granada. Recuperado de http://masteres.ugr.es/moea/pages/ curso201314/tfm1314/tfm-septiembre1314/memoriamasterantonio_lara_hormigo/!

Latorre, M., Aravena, P. Milos, P., \& García, M. (2010). Competencias habilitantes: un aporte para el reforzamiento de las trayectorias formativas universitarias. Calidad en la educación, 33, 275-301. Recuperado de https://www.cned.cl/sites/default/files/ revista/cse_articulo963.pdf 
Llanes Ordoñez, J., Figuera Gazo, P., \& Torrado Fonseca, M. (2017). Desarrollo de la empleabilidad y gestión personal de la carrera de graduados en pedagogía. Revista Española de Orientación y Psicopedagogía, 28(2), 46-60. https://doi.org/10.5944/reop. vol.28.num.2.2017.20118

Lluch Molins, L., Fernández-Ferrer, M., Pons Seguí, L., \& Cano García, E. (2017). Competencias profesionales de los egresados universitarios: estudio de casos en cuatro titulaciones. Revista Qurriculum, 30, 49-64. Recuperado de https://riull.ull.es/xmlui/ handle/915/6135

Lorente García, R. (2011). Configuración de las políticas europeas de formación profesional ante las nuevas demandas del mercado laboral. Revista de curriculum y formación del profesorado, 15 (2), 357- 369. Recuperado de http://www.ugr.es/ recfpro/ rev152COL11.pdf

Autor, \& Autor (2018). Validez de contenido y consistencia interna de un cuestionario sobre el proceso de inserción socio-laboral desde la mirada del universitario. Revista complutense de educación, 29(3), 739-756. Doi: http://dx.doi.org/10.5209/RCED.53721

Autor \& González Morga, N. (2018). Las competencias transversales en la universidad: propiedades psicométricas de un cuestionario. Educación XX1, 21(1), 231-262. Doi: 10.5944/educXX1.15662

Medina Rivilla, A., Domínguez Garrido, M.C., \& Sánchez Romero, C. (2013). Evaluación de las competencias de los estudiantes: modelos y técnicas para la valoración. Revista de Investigación Educativa, 31 (1), 239-255. Doi: http://dx.doi.org/10.6018/rie.31.1.157601

Medina, J. L., \& Jaruta, B. (2013). Enseñanza y aprendizaje en la Educación Superior. Madrid: Síntesis.

Michavila, F., Martínez, J. M., Martín-González, M., García-Peñalvo, F. J., \& Cruz-Benito, J. (2016). Barómetro de empleabilidad y empleo de los universitarios en España, 2015 (Primer informe de resultados). Madrid: Observatorio de Empleabilidad y Empleo Universitarios.

Mir Acebrón, A. (2007). Las competencias transversales en la Universidad Pompeu Fabra. La visión de los docentes y estudiantes de segundo ciclo. Revista de Docencia Universitaria, 1-16. Recuperado de http://revistas.um.es/redu/article/view/10641/10231

Montoro, M.A., Mora, E. M., \& Ortiz, M. (2012). Análisis de las competencias adquiridas en los estudios de Dirección de Empresas y su grado de aplicación en las prácticas en empresas. Revista complutense de educación, (23)1, 241-263.

Navaridas Nalda, F., Jiménez Trens, M.A. \& Fernández Ortiz, R. (2016). El aprendizaje de competencias en la Universidad: expectativas predictivas y niveles de confirmación de los estudiantes. Revista española de pedagogía, 74 (264) 337-356. Recuperado de https://dialnet.unirioja.es/servlet/articulo?codigo $=5436662$

Organización para la Cooperación y Desarrollo Económicos (OCDE). (2016). Automation and Independent Work in a Digital Economy. OECD.

OCDE (2015). Skills Strategy Informe de Diagnóstico. Informe diagnóstico de la estrategia de competencias de la OCDE: España. OCDE. Recuperado de https://skills.oecd.org/ developskills/.../Spain_Diagnostic_Report_Espagnol.

Organización Internacional del Trabajo (OIT). (2004). OIT (2004). Recomendación sobre el desarrollo de los recursos humanos: Educación, formación y aprendizaje permanente. Ginebra: OIT. Recuperado de: https://www.oitcinterfor.org/sites/default/files/edit/ docref/rec195.pdf 
Pagués Serra, C. \& Ripani, L. (2017). El empleo en la cuarta revolución industrial. IntegraciónE comercio, 42, 266-276. Recuperado de https://dialnet.unirioja.es/servlet/ articulo?codigo $=6174376$

Rodríguez Gómez, G., Ibarra Saiz, M.S., \& Cubero Ibañez, J. (2018). Competencias básicas relacionadas con la evaluación. Un estudio sobre la percepción de los estudiantes universitarios. Educación XXI, 21(1), 181-208. doi: http://dx.doi.org/10.5944/ educXX1.14457

Schumacker, R., \& Lomax, R., (2004). A beginner's guide to structural equation modeling ( $2^{\mathrm{a}}$ ed.). Mahwah, NJ: Lawrence Erlbaum Associates.

Tejada Fernández, J., \& Navío Gámez, A. (2005). El desarrollo y la gestión de competencias profesionales: una mirada desde la formación. Revista Iberoamericana de Educación, 37 (2), 1-16. Recuperado de https://rieoei.org/RIE/article/view/2719

Tynjälä, P., Slotte, V., Nieminen, J., Lonka, K., \& Olkinuora, E. ( 2006). From university to working life: Graduates' workplace skills in practice. En. P. Tynjälä, J. Väloimaa, and G. Boulton Lewis (ed.), Higher education and working life: Collaborations, confrontations and challenges, (pp.73-88). Amsterdam: Elsevier.

Van Breugel, G. (2017). Identifcation and anticipation of skill requirements. Instruments used by international institutions and developed countries. Recuperado de https:// www.oitcinterfor.org/sites/default/files/file_publicacion/Identifi_ant_skillsrequirements.pdf

Van der Heijde, C.M. (2014). Employability and Self-Regulation in Contemporary Careers. En M. Coetzee (Ed.). Psycho-social Career Metacapacities (pp.7-17). Londres: Springer.

Villardón, L. (coord.) (2015). Competencias genéricas en educación superior. Metodologías específicas para su desarrollo. Madrid: Narcea.

Fecha de recepción: 30 de septiembre de 2018.

Fecha de revisión: 1 de octubre de 2018.

Fecha de aceptación: 12 de octubre de 2018. 
